\title{
Erratum: Deciphering the combinatorial histone code
}

Joshua M Gilmore \& Michael P Washburn

Nat. Methods 4, 480-481 (2007).

For the work described in this News and Views piece, Craig Mizzen was an independent collaborator with members of the Kelleher lab. His role was not acknowledged, and we regret this editorial oversight.

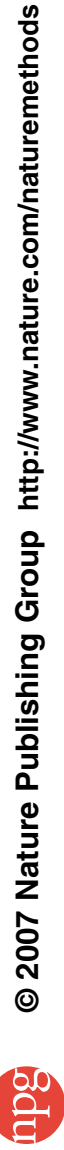

